\title{
Cluster expansion for the description of condensed state: crystalline cell approach
}

\author{
G.S. Bokun ${ }^{1}$, M.F. Holovko ${ }^{2}$ \\ 1 Belarusian State Technological University, 13a Sverdlov St., 220006 Minsk, Belarus \\ 2 Institute for Condensed Matter Physics of the National Academy of Sciences of Ukraine, \\ 1 Svientsitskii St., 79011 Lviv, Ukraine
}

Received May 2, 2018, in final form July 10, 2018

\begin{abstract}
A well-known cluster expansion, which leads to virial expansion for the free energy of low density systems, is modified in such a way that it becomes applicable to the description of condensed state of matter. To this end, the averaging of individual clusters over the states of an ideal gas is replaced by the averaging over the states of a non-correlated crystal using single-particle cell potentials. As a result, we arrive at the expansion of the partition function in correlations on the basis of single-particle functions corresponding to the multiplicative approximation. The cell potentials defining these functions are found from the condition of the minimum of the remainder in the constructed decomposition.
\end{abstract}

Key words: lattice models, cluster expansions, single-particle cell potential, free energy

PACS: 5.20.-y, 61.72jd, 64.30.-t, 65.40.-b

\section{Introduction}

The cellular theory, which is based on the structuring of the states of the system, has made it possible to solve a number of important problems in the physics of condensed state [1,2]. The lattice version of this theory has been used to address fundamental problems of statistical physics as well as to calculate specific properties of various systems [3, 4]. The development and introduction of nanomaterials has driven these approaches to be applied to the description of highly heterogeneous structures, which, in addition to large gradients of order parameter fields, are characterized by a heterogeneous state accompanied by phase transitions of various types [5, 6]. Here, as in the case of homogeneous systems, it seems effective to involve mean-field representations based on the use of cell potentials [7, 8].

The present paper is devoted to the development of the basis of this approach. To this end, a system of particles in the mean field of single-particle cell potentials is used as the reference system. The cell potentials determine the average forces acting on a particle fixed in a selected cell from the particles distributed in other molecular cells. Initially, only those states are taken into consideration where each cell of the system is occupied by one molecule or is vacant. The heterogeneity in the system is taken into account by the fact that the cell volumes are not the same. In order to calculate the partition function of the initial condensed system, the perturbation theory is used based on the expansions over the states of the reference system using generalized Mayer functions. These functions contain not only intermolecular interaction potentials but also the necessary mean potentials. The latter are determined from the condition for optimizing the deviation of the properties of the reference and the real systems. Furthermore, the thermodynamic consistency of the theory is studied at the level of calculation of the first derivatives with respect to thermodynamic parameters. The developed microscopic approach is generalized to the case of long-range interaction. A possibility to take account of a more complete set of occupation number values is discussed. 
The paper is arranged as follows. In section 2, the lattice model and the description of a corresponding reference system are presented. In order to take into account the inter-particle correlations in section 3 , we formulate the perturbation theory based on the expansions over the states of the reference system. In section 4, we formulate the optimal choice of a single-particle cell potentials needed for the description of the reference system. In section 5, the verification of thermodynamic self-consistency of the presented approach is considered at the level of calculation of the first derivatives with respect to thermodynamic parameters. In section 6 the generalization of the considered approach for the case of a more complete set of occupation numbers values and the generalization to the case of the systems with long-range interaction are discussed. We conclude in section 7

\section{The model and the reference system}

In this paper we consider a lattice model with the Hamiltonian

$$
H_{M}=\frac{1}{2} \sum_{i=1}^{M} \sum_{j(i)}^{Z} \Phi\left(q_{n_{i}}, q_{n_{j}}\right)+\sum_{i=1}^{M} \mu_{i} n_{i}
$$

where $M$ is the total number of lattice sites (i.e., cells in the system), $\sum_{j(i)}^{Z}$ denotes consecutive summation over all nodes surrounding the selected node $i$ taking into account $Z$ counted neighbors, $\mu_{i}$ is the chemical potential value in the node $i$. The classification of states considered is similar to that adopted in [9]. The variable $q_{n_{i}}$ determines the position of the particle $\left(n_{i}=1_{i}\right)$ or the vacancy $\left(n_{i}=0_{i}\right)$ in the cell $i$, $\Phi\left(q_{n_{i}}, q_{n_{j}}\right)$ is the interaction potential between two particles with coordinates $q_{n_{i}}$ and $q_{n_{j}}$. We suppose that this potential is short-ranged enough and can be taken, for instance, in the Lennard-Jones form [10].

In order to describe the considered model with the Hamiltonian (2.1), we apply the concept of the reference system widely used in the statistical theory of various condensed systems. Usually the reference system is a simplified version of the real condensed system. It should include the main features of the real model and should be described analytically with sufficient accuracy. For example, the simplest reference system is the model of the ideal gas. The application of this model leads to the virial density expansion for thermodynamic properties of real gases [11]. Another important reference system is the model of hard spheres, which has been successfully employed in the modern liquid state theory [10]. In this paper, to study the system with the Hamiltonian (2.1), we use the reference system that can be described by the Hamiltonian

$$
H_{0}=\sum_{i=1}^{M} \mu_{i} n_{i}+\sum_{i=1}^{M} \sum_{j(i)}^{Z} \phi_{j}\left(q_{n_{i}}\right)
$$

represented by single-particle cell potentials $\phi_{j}\left(q_{n_{i}}\right)$, which has the meaning of the potential of an external field whose source is located formally at the center of the cell $j . \phi_{j}\left(q_{n_{i}}\right)$ depends on the variables that determine the position of particles or vacancies in the cell $i$ and parametrically is a function of the quantities characterizing the average distribution of particles or vacancies in the system and its macroscopic state. In this case, $n=1$ corresponds to the distribution of particles and $n=0$ corresponds to that of vacancies.

The connection between the Hamiltonian $H_{M}$ and $H_{0}$ and the calculation of the single-particle potentials is discussed in the next sections. In this section we consider only the description of the reference system without elaboration of the single-particle cell potential $\phi_{j}\left(q_{n_{i}}\right)$.

We consider the case of an inhomogeneous system with inhomogeneity characterized by the field of mean occupation numbers

$$
\rho_{0}=\left\langle n_{i}\right\rangle_{0} .
$$

In order to simplify further expressions, we use abbreviations wherever possible, for example $U\left(q_{n_{i}}\right)=$ $U_{n_{i}}$ denote the potential acting on a particle or a vacancy in the position $q_{n_{i}}$. In order to take into account the variable number of particles and vacancies in the system, we consider both $q_{1_{i}}$ and $q_{0_{i}}$ as two states of a certain "virtual" particle. The first one corresponds to the position of a real particle and the second one to that of a vacancy. In this case, it is possible to replace fixed particles by arbitrary ones. 
This can be seen from the definition of $Z_{M}$

$$
Z_{M}=\sum_{N=0}^{M} \frac{1}{N !} Q_{N} \mathrm{e}^{-\mu N}
$$

Due to indiscernibility of particles, $N$ ! is reduced, which makes it possible to represent $Z_{M}^{(0)}$ of the reference system in the form

$$
Z_{M}^{(0)}=\sum_{n_{1}=0}^{1} \int_{\omega_{1}} \mathrm{~d} q_{n_{1}} \ldots \sum_{n_{i}=0}^{1} \int_{\omega_{i}} \mathrm{~d} q_{n_{i}} \ldots \sum_{n_{M}=0}^{1} \int_{\omega_{M}} \mathrm{~d} q_{n_{M}} \exp \left\{-\beta\left[\sum_{l=1}^{M}\left(\mu_{l} n_{l}+U_{n_{l}}\right)\right]\right\},
$$

where $\beta=1 /(k T), k$ is the Boltzmann constant, $T$ is the temperature, $\omega_{i}$ is the volume of the lattice cell $i$,

$$
U_{n_{l}}=\sum_{j(l)}^{Z} \phi_{j}\left(q_{n_{l}}\right) .
$$

In accordance with the representation (2.5), the particle distribution function over the volume of the system turns out to be factorized and can be written in the form

$$
D_{M}^{(0)}=\prod_{i=1}^{M} \rho\left(q_{n_{i}}\right)
$$

where

$$
\rho\left(q_{n_{i}}\right)=\exp \left[-\beta\left(\mu_{i} n_{i}+U_{n_{i}}\right)\right] .
$$

The normalization of this function, respectively, is represented by the expression

$$
Z_{M}^{(0)}=\prod_{i=1}^{M} z_{i}^{0}
$$

where

$$
\begin{gathered}
z_{i}^{0}=\sum_{n_{i}=0}^{1} \int_{\omega_{i}} \exp \left[-\beta\left(\mu_{i} n_{i}+U_{n_{i}}\right)\right] \mathrm{d} q_{n_{i}}, \\
z_{i}^{0}=Q_{0_{i}}+\mathrm{e}^{\beta \mu_{i}} Q_{1_{i}}, \\
Q_{n_{i}}=\int_{\omega_{i}} \exp \left[-\beta \sum_{j(i)}^{Z} \phi_{j}\left(q_{n_{i}}\right)\right] \mathrm{d} q_{n_{i}} .
\end{gathered}
$$

Based on equations 2.77- 2.12 , we write the expression for the normalized distribution function, which is necessary for averaging the magnitudes of the singlet, binary, and other types. Thus, denoting the normalized functions by a cap above the notations of respective functions, we write

$$
\widehat{D}_{M}=\prod_{i=1}^{M} \widehat{\rho}\left(q_{n_{i}}\right)
$$

where

$$
\widehat{\rho}\left(q_{n_{i}}\right)=\frac{\exp \left[-\beta\left(\mu_{i} n_{i}+U_{n_{i}}\right)\right]}{z_{i}^{0}} .
$$


We transform the above expressions based on the relation between $\mu_{i}$ and the mean values of the occupation numbers $\rho_{1_{i}}$ and $\rho_{0_{i}}$. Integrating (2.14) with respect to $q_{n_{i}}$, we find

$$
\rho_{n_{i}}=\frac{\mathrm{e}^{\beta \mu_{i} n_{i}} Q_{n_{i}}}{z_{i}^{0}} .
$$

From $(2.15)$ it follows that

$$
\frac{\rho_{1_{i}}}{\rho_{0_{i}}}=\frac{\mathrm{e}^{\beta \mu_{i}} Q_{1_{i}}}{Q_{0_{i}}} .
$$

The relation (2.16) allows us to write

$$
\mathrm{e}^{\beta \mu_{i}}=\frac{\rho_{1_{i}} Q_{0_{i}}}{\rho_{0_{i}} Q_{1_{i}}} .
$$

Substituting (2.17) into (2.11), we obtain

$$
z_{i}^{0}=Q_{0_{i}}+\frac{\rho_{1_{i}} Q_{0_{i}}}{\rho_{0_{i}}}=\frac{Q_{0_{i}}}{\rho_{0_{i}}} .
$$

The substitution of (2.17) and (2.18) allows us to write

$$
\widehat{\rho}\left(q_{n_{i}}\right)=\rho_{n_{i}} \frac{\exp \left[-\beta \sum_{j(i)}^{Z} \phi_{j}\left(q_{n_{i}}\right)\right]}{Q_{n_{i}}} .
$$

Let us consider the averaging that employs 2.13, for example the characteristics of a binary type $L\left(q_{n_{i}}, q_{n_{j}}\right)$ according to the definition

$$
\left\langle L_{i j}\right\rangle_{0}=\sum_{n_{1}=0}^{1} \sum_{n_{2}=0}^{1} \ldots \sum_{n_{m}=0}^{1} \int_{\omega_{1}} \widehat{\rho}\left(q_{n_{1}}\right) \ldots \int_{\omega_{j}} \widehat{\rho}\left(q_{n_{j}}\right) \ldots \int_{\omega_{M}} \widehat{\rho}\left(q_{n_{j}}\right) \ldots \int_{\omega_{m}} \widehat{\rho}\left(q_{n m}\right) L\left(q_{n_{i}} q_{n_{j}}\right) .
$$

Transposing the summation in 2.20 and taking into account the independence of integration variables and the normalized condition

$$
\sum_{n_{i}=0}^{1} \int_{\omega_{i}} \widehat{\rho}\left(q_{n_{i}}\right) \mathrm{d} q_{n_{i}}=\sum_{n_{i}=0}^{1} \rho_{n_{i}}=1
$$

we obtain

$$
\left\langle L_{i j}\right\rangle_{0}=\sum_{n_{i=0}}^{1} \sum_{n_{j}=0}^{1} \int_{\omega_{i}} \mathrm{~d} q_{n_{i}} \int_{\omega_{j}} \mathrm{~d} q_{n_{j}} L\left(q_{n_{i}}, q_{n_{j}}\right) \widehat{\rho}\left(q_{n_{i}}\right) \widehat{\rho}\left(q_{n_{j}}\right),
$$

or

$$
\begin{gathered}
\left\langle L_{i j}\right\rangle_{0}=\sum_{n_{i=0}}^{1} \sum_{n_{j}=0}^{1} L_{n_{i}, n_{j}} \rho_{n_{i}} \rho_{n_{j}}, \\
L_{n_{i}, n_{j}}=\int_{\omega_{i}} \mathrm{~d} q_{n_{i}} \int_{\omega_{j}} \mathrm{~d} q_{n_{j}} L\left(q_{n_{i}}, q_{n_{j}}\right) \widehat{F}_{11}\left(q_{n_{i}}\right) \widehat{F}_{11}\left(q_{n_{j}}\right) .
\end{gathered}
$$

The functions $\widehat{F}_{11}(q)$ in 2.24 correspond to singlet distribution functions $F_{11}$ in the approximation of the method of conditional distributions [9]

$$
\widehat{F}_{11}\left(q_{n_{i}}\right)=\frac{1}{Q_{n_{i}}} \exp \left[-\beta \sum_{j(i)}^{Z} \phi_{j}\left(q_{n_{i}}\right)\right] .
$$




\section{Perturbation theory}

In this section, we consider a perturbation scheme for the treatment of the remainder

$$
\Delta H_{M}=H_{M}-H_{0}=\frac{1}{2} \sum_{i=1}^{M} \sum_{j(i)}^{Z} \Delta \phi\left(q_{n_{i}}, q_{n_{j}}\right),
$$

where

$$
\Delta \phi\left(q_{n_{i}}, q_{n_{j}}\right)=\Phi\left(q_{n_{i}}, q_{n_{j}}\right)-\phi_{j}\left(q_{n_{i}}\right)-\phi_{i}\left(q_{n_{j}}\right) .
$$

Now, we represent the partition function of the original system in the form

$$
Z_{M}=Z_{M}^{(0)}\left\langle\mathrm{e}^{-\beta \Delta H_{M}}\right\rangle_{0},
$$

where $\langle\ldots\rangle_{0}$ is the averaging represented by the expression

$$
\langle L\rangle_{0}=\sum_{n_{1}=0}^{1} \ldots \sum_{n_{M}=0}^{1} \int_{\omega_{1}} \mathrm{~d} q_{n_{U}} \widehat{\rho}\left(q_{n_{U}}\right) \ldots \int_{\omega_{M}} \widehat{\rho}\left(q_{n_{M}}\right) \mathrm{d} q_{n_{M}} L .
$$

To calculate [3.3, a cumulant expansion [10, 12] is used, leading to an expansion in powers of the density if the averaging in (3.3) is performed over the states corresponding to an ideal gas [11]. In our case, the distribution characteristic of an ideal crystal is used as the reference system. This allows us to obtain a suitable description of the properties of a condensed system. The virial coefficients in this case become density functions. In other words, the transformation 3.3 is an expansion over the cluster correlations, although formally it has the form of an expansion over the Mayer functions. For the latter we use the renormalized Mayer functions of the form

$$
f\left(q_{n_{i}}, q_{n_{j}}\right)=\exp \left[-\beta \Delta \phi\left(q_{n_{i}}, q_{n_{j}}\right)\right]-1 .
$$

We should note that for vacancies $\Phi\left(q_{0_{i}}, q_{0_{j}}\right)=\Phi\left(q_{0_{i}}, q_{1_{j}}\right)=\Phi\left(q_{1_{i}}, q_{0_{j}}\right)=0$. However, the Mayer functions $f\left(q_{n_{i}}, q_{n_{j}}\right) \neq 0$ because in this case $\Delta \phi\left(q_{n_{i}}, q_{n_{j}}\right) \neq 0$.

Using the procedure of group expansion in 3.3 , we obtain

$$
\begin{aligned}
Z_{M} & =Z_{M}^{(0)} \exp \left[\frac{1}{2} \sum_{i=1}^{M} \sum_{j(i)}^{Z} \sum_{n_{i}=0}^{1} \sum_{n_{j}=0}^{1} \int_{\omega_{i}} \mathrm{~d} q_{n_{i}} \int_{\omega_{j}} \mathrm{~d} q_{n_{j}} \widehat{\rho}\left(q_{n_{i}}\right) \widehat{\rho}\left(q_{n_{j}}\right) f\left(q_{n_{i}}, q_{n_{j}}\right)\right. \\
& +\frac{1}{6} \sum_{i=1}^{M} \sum_{j(i)}^{Z} \sum_{l(i)}^{Z} \sum_{n_{i}=0}^{1} \sum_{n_{j}=0}^{1} \sum_{n_{l}=0}^{1} \int_{\omega_{i}} \mathrm{~d} q_{n_{i}} \int_{\omega_{j}} \mathrm{~d} q_{n_{j}} \int_{\omega_{l}} \mathrm{~d} q_{n_{l}} \widehat{\rho}\left(q_{n_{i}}\right) \widehat{\rho}\left(q_{n_{j}}\right) \widehat{\rho}\left(q_{n_{l}}\right) \\
& \left.\times f\left(q_{n_{i}}, q_{n_{j}}\right) f\left(q_{n_{j}}, q_{n_{l}}\right) f\left(q_{n_{i}}, q_{n_{l}}\right)+\ldots\right] .
\end{aligned}
$$

Or, due to representations 2.23 and 2.24 ,

$$
\begin{aligned}
Z_{M} & =Z_{M}^{(0)} \exp \left[\frac{1}{2} \sum_{i=1}^{M} \sum_{j(i)}^{Z} \sum_{n_{i}=0}^{1} \sum_{n_{j}=0}^{1} \rho_{n_{i}} \rho_{n_{j}} f_{n_{i} n_{j}}\right. \\
& \left.+\frac{1}{6} \sum_{i=1}^{M} \sum_{j(i)}^{Z} \sum_{l(i)}^{Z} \sum_{n_{i}=0}^{1} \sum_{n_{j}=0}^{1} \sum_{n_{l}=0}^{1} \rho_{n_{i}} \rho_{n_{j}} \rho_{n_{l}} f_{n_{i} n_{j} n_{l}}+\ldots\right],
\end{aligned}
$$


where

$$
\begin{aligned}
f_{n_{i} n_{j}} & =\int_{\omega_{i}} \mathrm{~d} q_{n_{i}} \int_{\omega_{j}} \mathrm{~d} q_{n_{j}} f\left(q_{n_{i}}, q_{n_{j}}\right) \widehat{F}_{11}\left(q_{n_{i}}\right) \widehat{F}_{11}\left(q_{n_{j}}\right), \\
f_{n_{i} n_{j} n_{l}} & =\int_{\omega_{i}} \mathrm{~d} q_{n_{i}} \int_{\omega_{j}} \mathrm{~d} q_{n_{j}} \int_{\omega_{l}} \mathrm{~d} q_{n_{l}} f\left(q_{n_{i}}, q_{n_{j}}\right) f\left(q_{n_{j}}, q_{n_{l}}\right) f\left(q_{n_{i}}, q_{n_{l}}\right) \\
& \times \widehat{F}_{11}\left(q_{n_{i}}\right) \widehat{F}_{11}\left(q_{n_{j}}\right) \widehat{F}_{11}\left(q_{n_{l}}\right) .
\end{aligned}
$$

From 3.7p, we have the cluster expansion for the free energy

$$
\begin{aligned}
F & =-k T \ln Z_{M}=-k T\left[\ln Z_{M}^{0}+\frac{1}{2} \sum_{i=1}^{M} \sum_{j(i)}^{Z} \sum_{n_{i}=0}^{1} \sum_{n_{j}=0}^{1} \rho_{n_{i}} \rho_{n_{j}} f_{n_{i} n_{j}}\right. \\
& \left.+\frac{1}{6} \sum_{i=1}^{M} \sum_{j(i)}^{Z} \sum_{l(i)}^{Z} \sum_{n_{i}=0}^{1} \sum_{n_{j}=0}^{1} \sum_{n_{l}=0}^{1} \rho_{n_{i}} \rho_{n_{j}} \rho_{n_{l}} f_{n_{i} n_{j} n_{l}}+\ldots\right] .
\end{aligned}
$$

\section{An optimal choice of a single-particle potential}

In order to apply the considered theory, we should specify the single particle potential. In this section we propose an optimal choice for this potential. This approach is in some sense similar to the problem of the connection between models with soft and hard core repulsions [13] which are successfully used in modern liquid state theory [14]. The equation defining the single-particle potentials of the reference system is determined from the self-consistent condition, which has several different formulations. One of them is connected with the extremum of the remainder in the expansion (3.6) due to the fact that the sum of all terms contained in (3.6) does not depend on the choice of single-particle potentials. Since a change of the potentials leads to redistribution of contributions of individual terms, the best choice would be the one with the maximum contribution of the terms that are taken into account. This is analogous to the requirement of the minimum susceptibility of the system to a virtual external field and leads to the condition

$$
\frac{\delta \ln Z_{M}}{\delta \phi_{k}\left(q_{n_{m}}\right)}=0 .
$$

Namely, the condition of the extremum of the part of the functional written in 3.77 corresponds simultaneously to the condition that the sum in brackets in (3.7) tends to zero. Let us consider the proof of the foregoing. We vary (3.7) over all the potentials in (2.6) assuming that they can all be independent of each other, which makes it possible to substantially simplify the procedure of transformations. Performing the variation with respect to an individual $\phi_{k}\left(q_{n_{m}}\right)$ and using 2.8) and 2.18), for the reference system part we obtain

$$
\begin{aligned}
\delta \ln z_{m}^{0} & =\frac{1}{z_{m}^{0}}\left\{\int_{\omega_{m}} \delta \phi_{k}\left(q_{0_{m}}\right) \exp \left[-\beta \sum_{j(m)}^{Z} \phi_{j}\left(q_{0_{m}}\right)\right] \mathrm{d} q_{0_{m}}\right. \\
& \left.+\int_{\omega_{m}} \delta \phi\left(q_{1_{m}}\right) \exp \left[-\beta \sum_{j(m)}^{Z} \phi_{j}\left(q_{1_{m}}\right)\right] \mathrm{e}^{\beta \mu_{m}} \mathrm{~d} q_{1_{m}}\right\} .
\end{aligned}
$$

Since the variation in $\delta \ln Z_{M}$ is satisfied for fixed $\mu_{i}$, we transform the second term in $Z_{M}$ into a form that contains explicit $\mu_{i}$.

$$
I_{m}=\frac{1}{2} \sum_{j(m)}^{Z} \sum_{n_{m}=0}^{1} \sum_{n_{j}=0}^{1} \rho_{n_{m}} \rho_{n_{j}} f_{n_{m} n_{j}}=\frac{1}{2} \sum_{j(m)}^{Z} \sum_{n_{m}=0}^{1} \sum_{n_{j}=0}^{1} \int_{\omega_{m}} \int_{\omega_{j}} \exp \left(-\beta\left\{\Phi\left(q_{n_{m}}, q_{n_{j}}\right)\right.\right.
$$




$$
\left.\left.+\sum_{s \neq m, j}\left[\phi_{s}\left(q_{n_{m}}\right)+\phi_{s}\left(q_{n_{j}}\right)\right]\right\}\right) \mathrm{e}^{\beta \mu_{m} n_{m}} \mathrm{e}^{\beta \mu_{j} n_{j}} \mathrm{~d} q_{n_{m}} \mathrm{~d} q_{n_{j}} \frac{1}{z_{m}^{0} z_{j}^{0}}-\frac{1}{2} .
$$

Let us write the expression for the variation of 4.3 with respect to $\phi_{k}\left(q_{n_{m}}\right)$

$$
\begin{aligned}
\delta \mathcal{I}_{m, k} & =\sum_{j \neq k, m} \sum_{n_{m}=0}^{1} \sum_{n_{j}=0}^{1} \int_{\omega_{m}} \int_{\omega_{j}} \delta \phi_{k}\left(q_{n_{m}}\right) \exp \left[-\beta \Phi\left(q_{n_{m}}, q_{n_{j}}\right)\right] \\
& \times \exp \left\{-\beta \sum_{s \neq m, j}\left[\phi_{s}\left(q_{m}\right)+\phi_{s}\left(q_{n_{j}}\right)\right]\right\} \mathrm{e}^{\beta \mu_{m} n_{m}} \mathrm{e}^{\beta \mu_{j} n_{j}} \mathrm{~d} q_{n_{m}} \mathrm{~d} q_{n_{j}} \frac{1}{z_{m}^{0} z_{j}^{0}} \\
& -\sum_{j(m)}^{Z} \sum_{n_{m}=0}^{1} \sum_{n_{j}=0}^{1} \int_{\omega_{m}} \int_{\omega_{j}} \exp \left\{-\beta \Phi\left(q_{n_{m}}, q_{n_{j}}\right)+\sum_{s \neq m, j}\left[\phi_{s}\left(q_{n_{m}}\right)+\phi_{s}\left(q_{n_{j}}\right)\right]\right\} \\
& \times \mathrm{e}^{\beta \mu_{m} n_{m}} \mathrm{e}^{\beta \mu_{j} n_{j}} \mathrm{~d} q_{n_{m}} \mathrm{~d} q_{n_{j}} \frac{1}{z_{m}^{0} z_{j}^{0}} \delta \ln z_{m}^{0} .
\end{aligned}
$$

The first sum on the right-hand side of equation (4.4) does not contain a term with $j=m$, which is convenient to add and subtract, which allows the sum (4.2) and (4.4) to be represented in a form that allows the separation of variables by cell numbers. Namely,

$$
\begin{aligned}
\delta \ln z_{m}^{0}+\delta \mathcal{I}_{m, k} & =\frac{1}{z_{m}^{0}} \sum_{n_{m}=0}^{1} \int_{\omega_{m}} \delta \phi_{k}\left(q_{n_{m}}\right) \exp \left[-\beta \sum_{j(m)}^{Z} \phi_{j}\left(q_{n_{m}}\right)\right] \mathrm{e}^{\beta \mu_{m} n_{m}} \mathrm{~d} q_{n_{m}} \\
& -\frac{1}{z_{m}^{0} z_{k}^{0}} \sum_{n_{m}=0}^{1} \sum_{n_{k}=0}^{1} \int_{\omega_{k}} \delta \phi_{k}\left(q_{n_{m}}\right) \mathrm{d} q_{n_{m}} \int_{\omega_{k}} \mathrm{~d} q_{n_{k}} \exp \left[-\beta \Phi\left(q_{n_{m}}, q_{n_{k}}\right)\right] \\
& \times \exp \left\{-\beta \sum_{s \neq m, k}\left[\phi_{s}\left(q_{n_{m}}\right)+\phi_{s}\left(q_{n_{k}}\right)\right]\right\} \exp \left[\beta\left(\mu_{m} n_{m}+\mu_{k} n_{k}\right)\right] \\
& +\sum_{j \neq m} A_{m, j}=0 .
\end{aligned}
$$

In the relation (4.5), the term $A_{m, j}$ is the symmetrized part of $\delta \mathcal{I}_{m, k}$ determined from equation (4.4). So, for $A_{m, j}$ we can write

$$
\begin{aligned}
A_{m, j} & =\frac{1}{z_{m}^{0} z_{j}^{0}} \sum_{n_{m}=0}^{1} \sum_{n_{j}=0}^{1} \mathrm{e}^{\beta\left(\mu_{m} n_{m}+\mu_{j} n_{j}\right)} \int_{\omega_{m}} \int_{\omega_{j}}\left[\delta \phi_{k}\left(q_{n_{m}}\right)-\delta \ln z_{m}^{0}\right] \\
& \times \exp \left[-\beta \Phi\left(q_{n_{m}}, q_{n_{j}}\right)\right] \exp \left\{-\beta \sum_{s \neq m, j}\left[\phi_{s}\left(q_{n_{m}}\right)+\phi_{s}\left(q_{n_{j}}\right)\right]\right\} \mathrm{d} q_{n_{m}} \mathrm{~d} q_{n_{j}} .
\end{aligned}
$$

Subsequent separation of variables makes it possible to obtain an equation for the required potentials $\phi_{j}\left(q_{n_{i}}\right)$. Since both $\delta \phi_{k}\left(q_{1_{m}}\right)$ and $\delta \phi_{k}\left(q_{0_{m}}\right)$ are independent, after simplifications of equations $(4.6)$, we obtain a system of defining equations

$$
\exp \left[-\beta \phi_{k}\left(q_{n_{m}}\right)\right]=\frac{1}{z_{k}^{0}} \sum_{n_{k}=0}^{1} \mathrm{e}^{\beta \mu_{k} n_{k}} \int_{\omega_{k}} \mathrm{~d} q_{n_{k}} \exp \left\{-\beta\left[\Phi\left(q_{n_{m}}, q_{n_{k}}\right)+\sum_{s \neq m, k}^{Z} \phi_{s}\left(q_{n_{k}}\right)\right]\right\} .
$$

Equations (4.7) can be rewritten in another form, namely when the density field is used as a variable that defines the system. Substituting 2.15 into 4.7 we arrive at a description

$$
\exp \left[-\beta \phi_{k}\left(q_{n_{m}}\right)\right]=\sum_{n_{k}=0}^{1} \frac{\rho_{n_{k}}}{Q_{n_{k}}} \int_{\omega_{k}} \mathrm{~d} q_{n_{k}} \exp \left\{-\beta\left[\Phi\left(q_{n_{m}}, q_{n_{k}}\right)+\sum_{s \neq m, k}^{Z} \phi_{s}\left(q_{n_{k}}\right)\right]\right\} \text {. }
$$


Equations 4.7) and (4.8) define the single-particle cell potentials under the condition that the twovertex diagrams in the cluster expansion of the original partition function (3.6) are equal to zero. As was shown in [15], this condition leads to the results that are equivalent to the quasichemical (or BetherPeierls) approximation. The inclusion of three-vertex diagrams in the equations (4.7) or (4.8) is also possible but it would lead to more complicated equations and, therefore, we will not consider it here.

Now, we show that when equation (4.7) is satisfied, each term $A_{j}$ in 4.5 turns out to be zero, which indicates that the separation constant of the variables in 4.5 chosen to be zero is correct.

Thus, substituting 4.7) into 4.6, we obtain

$$
A_{m, j}=\frac{1}{z_{m}^{0}} \sum_{n_{m=0}}^{1} \mathrm{e}^{\beta \mu_{m} n_{m}} \int_{\omega_{m}}\left[\delta \phi_{k}\left(q_{n_{m}}\right)-\delta \ln z_{m}^{0}\right] \exp \left[-\beta \sum_{s \neq m} \phi_{s}\left(q_{n_{m}}\right)\right] \mathrm{d} q_{n_{m}} .
$$

Using the definitions (2.11) and (2.12) in (4.9), we obtain

$$
A_{m, j}=\frac{1}{z_{m}^{0}} \sum_{n_{m=0}}^{1} \mathrm{e}^{\beta \mu_{m} n_{m}} \int_{\omega_{m}} \delta \phi_{k}\left(q_{n_{m}}\right) \exp \left[-\beta \sum_{s \neq m} \phi_{s}\left(q_{n_{m}}\right)\right] \mathrm{d} q_{n_{m}}-\delta \ln z_{m}^{0}
$$

In turn, it is clear from (4.2) that the first sum in 4.10 is identical to the variation of the second term, which proves that $A_{j}=0$ when choosing single-particle potentials satisfying equation (4.7). As already noted for specific calculations, (4.7) is preferable in the form (4.8) because this option allows for an implicit relationship between the chemical potential and the density to be replaced by an explicit one. Namely, for the chosen $\rho_{n_{i}}$ the solution (4.8) is found with (2.12) taken into account. Then, due to 2.17, the chemical potential and the free energy of the system are determined. To calculate the latter, it is convenient to use the relation

$$
-\beta F=\sum_{i=1}^{M}\left(\ln z_{i}^{0}-\rho_{1_{i}} \mu_{i}\right)
$$

Taking into account equation 2.17, expression 4.11) is represented in the form

$$
-\beta F=\sum_{i=1}^{M}\left[\ln z_{i}^{0}-\rho_{1_{i}} \ln \left(\frac{\rho_{1_{i}} Q_{0_{i}}}{\rho_{0_{i}} Q_{1_{i}}}\right)\right] .
$$

As a result, it follows from equation 4.7 that

$$
\sum_{n_{j}=0}^{1} \rho_{n_{j}} f_{n_{i} n_{j}}=0
$$

In order to prove 4.13 , we multiply equation 4.8 by

$$
\frac{\rho_{n_{m}}}{Q_{n_{m}}} \exp \left[-\beta \sum_{s \neq m} \phi_{s}\left(q_{n_{m}}\right)\right] \text {. }
$$

After integration, we obtain

$$
1=\sum_{n_{m}=0}^{1} \sum_{n_{k}=0}^{1} \rho_{n_{m}} \rho_{n_{k}} \int_{\omega_{m}} \int_{\omega_{k}} \exp \left[-\beta \Delta \phi\left(q_{n_{m}}, q_{n_{k}}\right)\right] \widehat{F}_{11}\left(q_{n_{m}}\right) \widehat{F}_{11}\left(q_{n_{k}}\right) \mathrm{d} q_{n_{m}} \mathrm{~d} q_{n_{k}} .
$$

The identity obtained with the definition of (3.8) proves the validity of equation (4.13). Hence, it follows that when the potentials are determined from $(4.8)$, the results of all three approaches are the same. Due to 4.13, $\ln z_{i}$ coincides with $\ln z_{i}^{0}$ which is defined by the relation 2.18 . 
Substituting 2.18) into the formula (4.12), we obtain an expression for the free energy of the system in the form

$$
-\beta F=\sum_{i=1}^{M}\left(\ln Q_{0_{i}}-\ln \rho_{0_{i}}+\rho_{1_{i}} \ln \rho_{0_{i}}+\rho_{1_{i}} \ln Q_{1_{i}}-\rho_{1_{i}} \ln \rho_{1_{i}}-\rho_{1_{i}} \ln Q_{0_{i}}\right)
$$

or

$$
-\beta F=\sum_{i=1}^{M}\left(\rho_{0_{i}} \ln Q_{0_{i}}+\rho_{1_{i}} \ln Q_{1_{i}}-\rho_{0_{i}} \ln \rho_{0_{i}}-\rho_{1_{i}} \ln \rho_{1_{i}}\right) .
$$

Such a representation of the right-hand side of equation 4.16 corresponds to a configurational integral in the form

$$
Q_{N}=Q_{N}^{0}=\sum_{i=1}^{M} \frac{Q_{1_{i}}^{\rho_{1_{i}}} Q_{0_{i}}^{\rho_{0_{i}}}}{\rho_{1_{i}}^{\rho_{1_{i}}} \rho_{0_{i}}^{\rho_{0_{i}}}}
$$

\section{Verification of thermodynamic self-consistency of the theory}

The expression for $Q_{N}^{0}$ in the form 4.17 can be obtained using the Hamiltonian

$$
H_{N}^{0}=\sum_{i=1}^{M} \sum_{j(i)}^{Z} \phi_{j}\left(q_{n_{i}}\right)
$$

To this end, it is necessary to consider the states corresponding to (5.1) based on the methods for forming local equilibrium distributions [16]. We show that the conditions (4.8), when the contributions of the third and the subsequent virial coefficients are not taken into account, give the identical $\rho_{i}$ determined by formulae

$$
\mu_{i}=\frac{\partial F}{\partial \rho_{i}}
$$

and

$$
\rho_{1_{i}}=\frac{\partial \ln Z_{M}^{0}}{\partial\left(\beta \mu_{i}\right)}
$$

In order to verify the thermodynamic consistency of the theory, we first consider the validity of equation 5.3. Using 2.18, we obtain

$$
\begin{aligned}
\rho_{1_{i}} & =\frac{\partial \ln \left(Q_{0_{i}}+\mathrm{e}^{\beta \mu_{i}} Q_{1_{i}}\right)}{\partial\left(\beta \mu_{i}\right)}+\sum_{k \neq i}^{M} \frac{\partial \ln z_{k}^{0}}{\partial\left(\beta \mu_{i}\right)} \\
& =\frac{1}{\left(Q_{0_{i}}+\mathrm{e}^{\beta \mu_{i}} Q_{1_{i}}\right)}\left[\mathrm{e}^{\beta \mu_{i}} Q_{1_{i}}+\frac{\partial Q_{0_{i}}}{\partial\left(\beta \mu_{i}\right)}+\frac{\partial Q_{1_{i}}}{\partial\left(\beta \mu_{i}\right)}\right]+\sum_{k \neq 1}^{M} \frac{\partial \ln z_{k}^{0}}{\partial\left(\beta \mu_{i}\right)} \\
& =\rho_{1_{i}}+\frac{1}{\left(Q_{0_{i}}+\mathrm{e}^{\beta \mu_{i}} Q_{1_{i}}\right)}\left[\frac{\partial}{\partial\left(\beta \mu_{i}\right)} Q_{0_{i}}+\mathrm{e}^{\beta \mu_{i}} \frac{\partial Q_{1_{i}}}{\partial\left(\beta \mu_{i}\right)}\right]+\sum_{k \neq i}^{M} \frac{\partial \ln z_{k}^{0}}{\partial\left(\beta \mu_{i}\right)} .
\end{aligned}
$$

It follows from (5.4) that (4.7) must satisfy the additional condition

$$
\frac{\partial}{\partial\left(\beta \mu_{i}\right)} Q_{0_{i}}+\mathrm{e}^{\beta \mu_{i}} \frac{\partial Q_{1_{i}}}{\partial\left(\beta \mu_{i}\right)}=0
$$


Then, $\rho_{1_{i}}$ calculated from the formulae $(5.3)$ and $(2.16)$ will coincide. To prove $(5.5)$ we differentiate the condition 4.7 with respect to $\beta \mu_{m}$

$$
\begin{aligned}
& \frac{\partial}{\partial\left(\beta \mu_{m}\right)}\left[-\beta \phi_{k}\left(q_{n_{m}}\right)\right] \exp \left[-\beta \phi_{k}\left(q_{n_{m}}\right)\right]=-\frac{1}{z_{k}^{0}} \frac{\partial \ln z_{k}^{0}}{\partial\left(\beta \mu_{m}\right)} \sum_{n_{k}=0}^{1} \exp \left(\beta \mu_{k} n_{k}\right) \\
& \times \int_{\omega_{k}} \mathrm{~d} q_{n_{k}} \exp \left\{-\beta\left[\Phi\left(q_{n_{m}}, q_{n_{k}}\right)+\sum_{s \neq m, k}^{Z} \phi_{s}\left(q_{n_{k}}\right)\right]\right\}+\frac{1}{z_{k}^{0}} \sum_{n_{k}=0}^{1} \exp \left(\beta \mu_{k} n_{k}\right) \\
& \times \int_{\omega_{k}}\left[\sum_{s=m, k}^{Z} \frac{\partial \phi_{s}\left(q_{n_{k}}\right)}{\partial\left(\beta \mu_{m}\right)}\right] \exp \left\{-\beta\left[\Phi\left(q_{n_{m}}, q_{n_{k}}\right)+\sum_{s \neq m, k}^{Z} \phi_{s}\left(q_{n_{k}}\right)\right]\right\} \mathrm{d} q_{n_{k} .} .
\end{aligned}
$$

Multiplying 4.10 by $\frac{1}{z_{m}^{0}} \exp \left[-\beta \sum_{s \neq k, m} \phi_{s}\left(q_{n_{m}}\right)\right]$, after integration over $q_{n_{m}}$ we obtain

$$
\begin{aligned}
& \frac{1}{z_{m}^{0}} \int_{\omega_{m}} \frac{\partial}{\partial\left(\beta \mu_{m}\right)}\left[-\beta \phi_{k}\left(q_{n_{m}}\right)\right] \exp \left[-\beta \sum_{s \neq k}^{Z} \phi_{s}\left(q_{n_{m}}\right)\right] \mathrm{d} q_{n_{m}} \\
& =-\frac{1}{z_{m}^{0}} \frac{\partial \ln z_{k}^{0}}{\partial\left(\beta \mu_{m}\right)} \int_{\omega_{m}} \exp \left[-\beta \sum_{s \neq k}^{Z} \phi_{s}\left(q_{n_{m}}\right)\right] \mathrm{d} q_{n_{m}} \\
& +\frac{1}{z_{k}^{0} z_{m}^{0}} \sum_{n_{k}=0}^{1} \exp \left(\beta \mu_{k} n_{k}\right) \int_{\omega_{k}} \int_{\omega_{m}} \mathrm{~d} q_{n_{m}} \mathrm{~d} q_{n_{k}} \exp \left[-\beta \Phi\left(q_{n_{k}}, q_{n_{m}}\right)\right] \\
& \times\left[-\beta \sum_{s \neq m, k}^{Z} \frac{\partial \phi_{s}\left(q_{n_{k}}\right)}{\partial\left(\beta \mu_{m}\right)}\right] \exp \left[-\beta \sum_{s \neq k}^{Z} \phi_{s}\left(q_{n_{m}}\right)\right]
\end{aligned}
$$

Multiplying 5.7 by $\sum_{n_{m}}^{1} \mathrm{e}^{-\beta \mu_{m} n_{m}}$ and taking into account 2.11 and 4.7, we have

$$
\begin{aligned}
& \frac{2}{z_{m}^{0}} \sum_{n_{m}=0}^{1} \mathrm{e}^{-\beta \mu_{m} n_{m}} \int_{\omega_{m}} \frac{\partial}{\partial\left(\beta \mu_{n}\right)}\left[-\beta \phi_{k}\left(q_{n_{m}}\right)\right] \exp \left[-\beta \sum_{s \neq k}^{Z} \phi_{s}\left(q_{n_{m}}\right)\right] \mathrm{d} q_{m} \\
& =-\frac{\partial \ln z_{k}^{0}}{\partial\left(\beta \mu_{m}\right)}+\frac{1}{z_{k}^{0}} \sum_{n_{k}=0}^{1} \exp \left(\beta \mu_{k} n_{k}\right) \int_{\omega_{k}}\left[-\beta \sum_{S \neq k}^{Z} \frac{\partial \phi_{s}\left(q_{n_{k}}\right)}{\partial\left(\beta \mu_{m}\right)}\right] \exp \left[-\beta \sum_{s \neq k}^{Z} \phi_{s}\left(q_{n_{m}}\right)\right] \mathrm{d} q_{n_{k}} .
\end{aligned}
$$

As a result,

$$
\begin{aligned}
& \frac{2}{z_{m}^{0}} \sum_{n_{m}=0}^{1} \mathrm{e}^{-\beta \mu_{m} n_{m}} \int_{\omega_{m}} \frac{\partial}{\partial\left(\beta \mu_{m}\right)}\left[-\beta \phi_{k}\left(q_{n_{m}}\right)\right] \exp \left[-\beta \sum_{s \neq k}^{Z} \phi_{s}\left(q_{n_{m}}\right)\right] \mathrm{d} q_{n_{m}} \\
& =\frac{\partial \ln z_{k}^{0}}{\partial\left(\beta \mu_{m}\right)}+\frac{1}{z_{k}^{0}} \sum_{n_{k}=0}^{1} \exp \left(\beta \mu_{k} n_{k}\right) \frac{\partial Q_{n_{k}}}{\partial}\left(\beta \mu_{m}\right)=0 .
\end{aligned}
$$

Performing the summation in 5.9 with respect to $k \neq m$, we find

$$
\sum_{n_{m}=0}^{1} \mathrm{e}^{-\beta \mu_{m} n_{m}} \frac{\partial Q_{n_{m}}}{\partial\left(\beta \mu_{m}\right)}=0
$$


Likewise, we show that although in accordance with 4.7$)$ each $\phi_{s}\left(q_{n_{k}}\right)$ depends on the chemical potentials in the entire range of the $k$-th node, it turns out that

$$
\frac{\partial z_{m}^{0}}{\partial\left(\beta \mu_{l}\right)}=0
$$

for $l \neq m$.

In combination with the condition 5.10 , this will ultimately prove the thermodynamic consistency of the theory when calculating the thermodynamic potentials $F$ and $\Omega$ and their first derivatives, determined by formulae (2.17) and (5.3). The proof of the condition $(5.11)$ is carried out similarly to $(5.5)$.

In order to reduce the transformations and make them more transparent, we write a relation analogous to 5.6 in the form

$$
\begin{aligned}
& \left\{\frac{\partial}{\partial\left(\beta \mu_{s}\right)}\left[-2 \beta \phi_{k}\left(q_{n_{m}}\right)\right]\right\} \exp \left[-\beta \phi_{k}\left(q_{n_{m}}\right)\right]=-\frac{\partial \ln z_{k}^{0}}{\partial\left(\beta \mu_{s}\right)} \exp \left[-\beta \phi_{k}\left(q_{n_{m}}\right)\right] \\
& +\frac{1}{z_{k}^{0}} \sum_{n_{k}=0}^{1} \exp \left(\beta \mu_{k} n_{k}\right) \int_{\omega_{k}}\left[\sum_{s \neq m}^{Z} \frac{\partial \beta \phi_{s}\left(q_{n_{k}}\right)}{\partial\left(\beta \mu_{s}\right)}\right] \exp \left\{-\beta\left[\Phi\left(q_{n_{m}}, q_{n_{k}}\right)\right.\right. \\
& \left.\left.+\sum_{s \neq m, k}^{Z} \phi_{s}\left(q_{n_{k}}\right)\right]\right\} \mathrm{d} q_{n_{k}} .
\end{aligned}
$$

Let us apply the following operation to relation 5.12

$$
\widehat{\pi}=\sum_{k \neq m}^{Z} \frac{1}{z_{m}^{0}} \sum_{n_{m}}^{1} \exp \left(-\beta \mu_{m} n_{m}\right) \int_{\omega_{m}} \exp \left[-\beta \sum_{s \neq k}^{Z} \phi_{s}\left(q_{n_{m}}\right)\right] \mathrm{d} q_{n_{m}} \ldots
$$

writing equation 5.12 in a symbolic form

$$
A=B+C
$$

When converting the left-hand side of expression (5.14), the summation sign with respect to $k \neq m$ is put under the integration sign with respect to $\omega_{m}$, and taking into account 2.12), we write the result in the form

$$
\widehat{\pi} A=2 \frac{1}{z_{m}^{0}} \sum_{n_{m}=0}^{1} \exp \left(-\beta \mu_{m} n_{m}\right) \frac{\partial Q_{n_{m}}}{\partial\left(\beta \mu_{s}\right)} .
$$

Since in 5.15 $s \neq m$ and $\mu_{m}$ and $\mu_{s}$ are independent variables, we switch the summation over $n_{m}$ and differentiation with respect to $\mu_{s}$, which due to 2.11 allows us to write

$$
\widehat{\pi} A=2 \frac{\partial \ln z_{m}^{0}}{\partial\left(\beta \mu_{s}\right)} .
$$

The result of the transformation of the expression $B$ is equal to 1. It can be obtained if the operation (5.3) is applied to $\exp \left[-\beta \phi_{k}\left(q_{n_{m}}\right)\right]$ taking the constant as the sign of operation 5.13. As a result, direct application of $\widehat{\pi}$ gives

$$
\widehat{\pi} B=-\frac{\partial \ln z_{k}^{0}}{\partial\left(\beta \mu_{s}\right)} .
$$

To transform the expression $C$, we switch the summation and integration operations in (5.13), place the operator $\widehat{\pi}$ under the integration sign with respect to $\omega_{k}$ and take into account that according to 4.7

$$
\widehat{\pi} \exp \left\{-\beta\left[\Phi\left(q_{n_{k}}, q_{n_{m}}\right)\right]\right\}=\exp \left[-\beta \phi_{m}\left(q_{n_{k}}\right)\right] .
$$


Then, $\widehat{\pi} C$ becomes of the form

$$
\widehat{\pi} C=\frac{1}{z_{k}^{0}} \sum_{n_{k}=0}^{1} \exp \left(\beta \mu_{k} n_{k}\right) \frac{\partial \ln Q_{k}}{\partial\left(\beta \mu_{s}\right)} .
$$

Taking 2.11) into account, condition (5.19) gives

$$
\widehat{\pi} C=\frac{\partial \ln z_{k}^{0}}{\partial\left(\beta \mu_{s}\right)} .
$$

The results (5.17) and 5.20 show that according to 5.14$)$, the expression $(5.16)$ is identically equal to zero. This, in turn, proves the validity of 5.12 .

Similarly to the consistency of (5.3) and 2.15) proved by derivations (5.4)- 5.20 , , one can show that (5.2) is consistent with (2.16). To this end, it is necessary to perform transformations similar to those performed for (4.7) over (4.8), differentiating each of the equations (5.4)-(5.20) with respect to thermodynamic variables $\rho_{n_{i}}$ but not as it was before with respect to $\mu_{s}$. Further analysis of the consistency problem involves comparing the results of calculating the second derivatives of thermodynamic potentials, and will be considered separately.

\section{Discussion}

In this paper, for a lattice model with the Hamiltonian (2.1) and a pair interaction potential $\Phi\left(q_{n_{i}}, q_{n_{j}}\right)$ we formulate a reference system with the Hamiltonian $(2.2)$ and a single-particle cell potential $\phi_{j}\left(q_{n_{i}}\right)$. The potential $\phi_{j}\left(q_{n_{i}}\right)$ can be interpreted as the mean potential exerted by a particle in the lattice cell $\omega_{j}$ on a particle in the lattice cell $\omega_{i}$. It is shown that the system with the single-cell potential $\phi_{j}\left(q_{n_{i}}\right)$ reduces to the description of a Fermi-like lattice model in an external field. Using this system as the reference system and renormalized Mayer functions in the form 3.5 with $\Delta \phi\left(q_{n_{i}}, q_{n_{j}}\right)$ in the form 3.2 , the generalized cluster expansion for the free energy of the considered system is obtained. The cell potentials are calculated from the condition of the minimum difference of thermodynamic properties of the systems with the Hamiltonians (2.1) and 2.2. Such a procedure is considered under the condition that the two-vertex diagrams in the cluster expansion of the partition function for the system with the Hamiltonian (2.1) are equal to zero. As a result, for the single-particle cell potential, a system of equations (4.7) was obtained. This system can also be presented in the form of equation (4.8). As it was noted previously in the reference [15], such a description is equivalent to the quasichemical approximation. We should note that the descriptions of the considered system in the framework of the Hamiltonians (2.1) and (2.2) are exactly equivalent only in the case when all the terms in the cluster expansion (3.10) are taken into account at the calculation of the single-particle potentials. There are two principal differences between the considered equation 4.7 and the corresponding equations in traditional approaches such as the mean field approximation formulated in the framework of the field theoretical approach [17, 18] or the density functional approach [19] well developed for non-lattice fluid systems. The first difference is connected with the presence of the interparticle potential $\Phi\left(q_{n_{i}}, q_{n_{j}}\right)$ in equation (4.7) in the exponential form. The second one is connected with the inequality $s \neq m, k$ in the exponent of equation (4.7). It means that instead of the singlet distribution function $\hat{F}_{11}\left(q_{n_{k}}\right)$, which usually appears in the mean field approximation, in the approach considered, the function $\hat{F}_{11}\left(q_{n_{k}}\right) \exp \left[\beta \phi_{m}\left(q_{n_{k}}\right)\right]$ appears. This is the result of the peculiarity of the Mayer functions in the form 3.5 with $\Delta \phi\left(q_{n_{i}}, q_{n_{j}}\right)$ in the form 3.2 . If we neglect the condition $s \neq m, k$ due to the renormalization condition (2.21), the equation (4.7) can be rewritten in the form

$$
\mathrm{e}^{-\beta \phi_{k}\left(q_{n_{m}}\right)}=1+\sum_{n_{k}=0}^{1} \rho_{n_{k}} \int \mathrm{d} q_{n_{k}}\left\{\exp \left[-\beta \Phi\left(q_{n_{m}}, q_{n_{k}}\right)\right]-1\right\} \hat{F}_{11}\left(q_{n_{k}}\right)
$$


which after linearization of the exponents $\exp \left[-\beta \phi_{k}\left(q_{n_{m}}\right)\right]$ and $\exp \left[-\beta \Phi\left(q_{n_{m}}, q_{n_{k}}\right)\right]$ leads to a traditional form for the mean field approximation

$$
\phi_{k}\left(q_{n_{m}}\right)=\sum_{n_{k}=0}^{1} \rho_{n_{k}} \int \mathrm{d} q_{n_{k}} \Phi\left(q_{n_{m}}, q_{n_{k}}\right) \hat{F}_{11}\left(q_{n_{k}}\right) .
$$

The equation 4.77 describes the single-particle cell potentials $\phi_{k}\left(q_{1_{m}}\right)$ for real particles, but for vacancies it is more of a problem. This problem is similar to the description of solvophobic interaction in the theory of solutions [20, 21] and for a correct description of single-particle cell potential $\phi_{k}\left(q_{0_{m}}\right)$ for vacancies, at least three-vertex diagrams in the equation (4.7) should be included. In the twovertex diagram approximation for $\phi_{k}\left(q_{0_{m}}\right)$ due to inequality $s \neq m, k$ there appears only some constant corresponding to the change of the chemical potential due to the creation of a vacancy.

The theory presented here is easily generalized when it is necessary to take into account a larger number of possible states. This is achieved by expanding the possible values of the occupation numbers, when $n_{i}=0,1,2 \ldots$ In this case, for every microconfiguration given by the set of values $\left\{n_{1}, \ldots, n_{m}\right\}$, the expression for the Hamiltonian is completely preserved in the form (2.1). So, for example, for $n=0,1,2$ in all formulae $(2.2)-(5.20)$ one should remember that

$$
\Phi\left(q_{n_{i}}, q_{n_{j}}\right)=\left\{\begin{array}{l}
0, n_{i}, n_{j}=0, \\
h\left(q_{i}, q_{j}\right), n_{i}, n_{j}=1, \\
h\left(q_{i}, q_{j}\right)+h\left(q_{i}, q_{j}^{\prime}\right), n_{i}, n_{j}=2, \\
h\left(q_{i}, q_{j}\right)+h\left(q_{i}, q_{j}^{\prime}\right)+h\left(q_{i}^{\prime}, q_{j}\right)+h\left(q_{i}^{\prime}, q_{j}^{\prime}\right), n_{i}, n_{j}=4 .
\end{array}\right.
$$

Here, $h\left(q_{i}, q_{j}\right)$ is the intermolecular interaction potential of two particles in positions $q_{i}$ and $q_{j}$, when $\left(q_{i}, q_{i}^{\prime}\right) \in \omega_{i},\left(q_{j}, q_{j}^{\prime}\right) \in \omega_{j}$.

In addition to taking into account (6.3) in all expressions (2.2)-(5.20), the summation over $n=0,1$ must be extended to the case $n=0,1,2$, additionally taking into account that

$$
\int_{\omega_{i}} \mathrm{~d} q_{0_{i}}=\frac{1}{\omega_{i}} \int_{\omega_{i}} \mathrm{~d} q_{i}, \quad \int_{\omega_{i}} \mathrm{~d} q_{1_{i}}=\int_{\omega_{i}} \mathrm{~d} q_{i}, \quad \int_{\omega_{i}} \mathrm{~d} q_{2_{i}}=\int_{\omega_{i}} \mathrm{~d} q_{i} \int_{\omega_{i}} \mathrm{~d} q_{i}^{\prime} .
$$

Thus, it is shown that the properties of a condensed system can be described by combining the model of an ideal crystal with a group expansion over the modified Mayer functions that impose correlations on the properties of an ideal crystal.

It seems justified to extend the developed approach to take into account long-range effects in essentially inhomogeneous media. Let us demonstrate the possibility of such propagation using the example when the inhomogeneity of the medium is described by different sizes of microcells, provided that in each of them there is one particle. Representing the energy of the system by short-range $\Phi(i, j)$ and long-range $V(i, j)$ potentials of pair interactions, respectively, for particles in positions $q_{i}, q_{j}$, we present the configurational integral $Q_{N}$ of the system in the form

$$
Q_{N}=Q_{N}^{0}\left\langle\exp \left[-\beta \sum_{i<j}^{N} V(i, j)\right] \prod_{i<j}^{N}[1+f(i, j)]\right\rangle_{0},
$$

where

$$
\begin{gathered}
f(i, j)=\exp \left\{-\beta[\Phi(i, j)]-\phi_{j}(i)-\phi_{i}(j)\right\}-1, \\
Q_{N}^{0}=\prod_{i=1}^{N} Q_{i}, \quad Q_{i}=\int_{v_{i}} \exp \left[-\beta \sum_{k \neq i} \phi_{k}(i)\right] \mathrm{d} q_{i} .
\end{gathered}
$$

$Q_{N}^{0}$ is the configurational integral of an ideal crystal, expressed through single-particle cell potentials of mean forces $\phi_{j}(i), f(i, j)$ is a renormalized Mayer function, the angle brackets $\langle\ldots\rangle_{0}$ denote averaging over 
the equilibrium states of the reference system. The subsequent cumulant expansion of the expression (2.2) with respect to the functions (2.3) allows one to write in the approximation of the second virial coefficient

$$
\begin{gathered}
\ln Q_{N}=\ln Q_{L}^{0}+\ln Q_{N}^{0}+\sum_{i, j}\langle f(i, j) g(i, j)\rangle_{0}+\ldots, \\
Q_{L}^{0}=\left\langle\exp \left[-\beta \sum_{i<j} V(i, j)\right]\right\rangle_{0}
\end{gathered}
$$

The averaging in relations 6.7) and 6.9) is realized by multiplying the unary distribution functions $F_{11}(i)$ and $F_{11}(j)$

$$
F_{11}(i)=\frac{1}{Q_{i}} \exp \left[-\beta \sum_{k \neq 1} \phi_{k}(i)\right]
$$

A special feature of relation (6.9) is that the renormalized Mayer function $f(i, j)$ is modulated here by a binary function, for which a consistent calculation scheme was developed in [10]. Applying the procedure of self-consistent calculation of the potentials $\phi_{j}(i)$ in accordance with the foregoing, we arrive at a closed system of equations of the form

$$
\exp \left[-\beta \phi_{j}(i)\right]=\frac{1}{Q_{j}} \int_{v_{j}} g(i, j) \exp [-\beta \Phi(i, j)] \times \exp \left[-\beta \sum_{k \neq i, j} \phi_{k}(j)\right] \mathrm{d} j .
$$

The system of equations (6.11) differs from the one used earlier because its kernel, in addition to the point short-range potential, contains a binary function for a system of particles with a Coulomb interaction, the expression for which has the form [10]

$$
\begin{aligned}
F_{2}(i, j) & =F_{11}(i) F_{11}(j) g(i, j), \\
g(i, j) & =\exp [-\beta u(i, j)],
\end{aligned}
$$

where $u(i, j)$ is a screening potential. Such a form solves the problem of the divergence of the integrals when calculating the free energy (6.7). As a result, long-range effects apply also to the renormalization of single-particle cell potentials. In expression (6.12), only the initial terms of the series are written corresponding to the Debye description of ion systems. A more complete representation for relation (6.12) follows when calculating additional terms of the distribution function $g(i, j)$ using collective variables [10]. A generalization of Debye screening to the system of mobile ions in lattice models was recently discussed in [4]. It leads to the change of the traditional inverse Debye length $\kappa$ to the new one $\kappa=\left[\beta e^{2} c(1-c) /\left(\varepsilon_{0} \varepsilon h^{3}\right)\right]^{1 / 2}$, where $e$ is the charge of a mobile ion, $\varepsilon_{0}$ is the dielectric permittivity of the vacuum, $\varepsilon$ is the relative dielectric permittivity of the medium, $c$ is the concentration of mobile ions, $h$ is the length of the cell.

The peculiarity of the equations in the case under consideration is due to the fact that the function $g(i, j)$ is determined by averaging not over the states of an ideal gas, but over the states of an ideal crystal. Accordingly, for example for $i=1, j=2$, the function $g(i, j)$ is defined by an expression of the form

$$
g(1,2)=\int_{v_{3}} \ldots \int_{v_{N}} \exp \left[-\beta \sum_{l<m=1}^{N} V(l, m)\right] F_{11}(3) F_{11}(4) \ldots F_{11}(N) \mathrm{d} 3 \mathrm{~d} 4 \ldots \mathrm{d} N .
$$

There are reasons to believe that the appearance of additional Gaussian in $(6.13)$ introduced by the functions $F_{11}$ will lead to an improvement in the convergence of the series 6.12 obtained in the framework of the collective variables method [10] since a similar procedure was successful in constructing the description using an effective potential [22]. 


\section{Conclusions}

A method for modifying the cluster virial expansion, which makes it possible to describe condensed state, is proposed. The method is based on replacing the averaging over the states of an ideal gas by the averaging over the states of an ideal crystal. The approach outlined differs from the well-known perturbation theory because the basis with respect to which the expansion is performed is not a part of the original Hamiltonian but is introduced independently. The basis distribution conveys the main features of the solid state of matter, where the motion of molecules is of an oscillatory nature with respect to the lattice sites. As a result, the Hamiltonian of the basic reference system is represented by the sum of single-particle cell mean force potentials. It is shown how the Mayer functions can be modified so that they would act as a small parameter for the subsequent expansion of the thermodynamic potential by cumulant expansions. From the condition of independence of the initial partition function on the introduced potentials of the mean forces, a system of integral equations determining the above potentials is obtained. The expression for the free energy functional is obtained with the two first terms of its expansion in correlations taken into account. It is shown that the obtained equations satisfy several optimization conditions for the parameters characterizing the properties of the reference system and the initial system. The thermodynamic consistency of various methods for calculating the thermodynamic characteristics of a condensed medium both in the canonical and in the grand canonical ensembles is proved. A possibility of using the developed approach to take into account not only short-range but also long-range interactions is shown.

\section{Acknowledgements}

This project has received funding from European Unions Horizon 2020 research and innovation programme under the Marie Skłodowska-Curie (grant agreement No 734276), the Belarusian Republican Foundation for Fundamental Research (grant No $\Phi 16 \mathrm{~K}-061$ ) and the State Fund for Fundamental Research of Ukraine (grant No Ф73/26-2017). We thank Ivan Kravtsiv and Dung di Caprio for the careful reading of the manuscript and useful comments.

\section{References}

1. Prigogine I., Bellemans A., Mathot V., The Molecular Theory of Solutions, North-Holland Publishing Company, Amsterdam, 1957.

2. Frenkel J.I., Kineticheskaya Teoriya Zhidkostei, AN SSSR, Moskva-Leningrad, 1945 (in Russian), [Kinetic Theory of Liquids, Dover Publications, Inc., New York, 1955].

3. Yukhnovskii I.R., Phase Transitions of the Second Order: Collective Variables Method, Word Scientific, Singapore, 1987.

4. Bokun G., di Caprio D., Holovko M., Vikhrenko V., J. Mol. Liq., 2018, 270, 183, doi $10.1016 /$ j.molliq.2018.03.123

5. Bisquert J., Vikhrenko V.S., J. Phys. Chem. B, 2004, 108, 2313, doi:10.1021/jp035395y

6. Ciach A., Góźdź W.T., Condens. Matter Phys., 2010, 13, 23603, doi 10.5488/CMP.13.23603.

7. Narkevich I.I., Physica A, 1982, 112, 167, doi 10.1016/0378-4371(82)90213-8

8. Di Caprio D., Badiali J.P., Holovko M., J. Phys. A: Math. Theor., 2009, 42, 214038, doi $10.1088 / 1751-8113 / 42 / 21 / 214038$

9. Rott L.A., Statistical Theory of Molecular Systems, Nauka, Moscow, 1979 (in Russian).

10. Yukhnovskii I.R., Holovko M.F., Statistical Theory of Classical Equilibrium Systems, Naukova Dumka, Kiev, 1980 (in Russian).

11. Van Kampen N.G., Physica, 1961, 27, 783, doi:10.1016/0031-8914(61)90097-0

12. Kubo R., J. Phys. Soc. Jpn., 1962, 17, 1100, doi:10.1143/JPSJ.17.1100

13. Andersen H.C., Weeks J.D., Chandler D., Phys. Rev. A, 1971, 4, 1597, doi 10.1103/PhysRevA.4.1597

14. Barker J.A., Henderson D., Rev. Mod. Phys., 1976, 48, 587, doi 10.1103/RevModPhys.48.587.

15. Argyrakis P., Groda Y.G., Bokun G.S., Vikhrenko V.S., Phys. Rev. E, 2001, 64, 066108, doi $10.1103 /$ PhysRevE.64.066108

16. Zubarev D.N, Nonequilibrium Statistical Thermodynamics, Nauka, Moscow, 1971 (in Russian). 
17. Di Caprio D., Stafiej J., Holovko M., Kravtsiv I., Mol. Phys., 2011, 109, 695,

doi $10.1080 / 00268976.2010 .547524$

18. Kravtsiv I., Patsahan T., Holovko M., di Caprio D., J. Chem. Phys., 2015, 142, 194708, doi 10.1063/1.4921242

19. Evans R., In: Fundamentals of Inhomogeneous Fluids, Henderson D. (Ed.), Marcel Dekker, New York, 1992, $85-175$.

20. Ronis D., Martina E., Deutch J.M., Chem. Phys. Lett., 1977, 46, 53, doi $10.1016 / 0009-2614(77) 85161-0$

21. Bandura A.V., Holovko M.F., Lvov S.N., J. Mol. Liq., 2018, 270, 52, doi $10.1016 /$ j.molliq.2018.01.015

22. Ma S.-K., Modern Theory of Critical Phenomena, Westview Press, New York, 1976.

\title{
Групове розвинення для опису конденсованих систем: підхід кристалічних комірок
}

\author{
Г.С. Бокун \\ 1 Білоруський державний технологічний університет, вул. Свердлова, 13а, 220006 Мінськ, Білорусь \\ 2 Інститут фізики конденсованих систем НАН України, вул. Свєнціцького, 1, 79011 Львів, Україна
}

Широко відоме групове розвинення, яке приводить до віріального розвинення для вільної енергії розріджених систем, модифіковано так, щоб його можна було застосовувати до конденсованого стану речовини. Для цього усереднення окремих кластерів по станах ідеального газу замінюється усередненням по станах некорельованого кристала, використовуючи коміркові одночастинкові потенціали. В результаті отримано розвинення статистичної суми по кореляціях на базисі одночастинкових функцій, які відповідають мультиплікативному наближенню. Ґраткові потенціали, що визначають вказані функції, знаходяться з умови мінімізації залишку в сконструйованому розвиненні.

Ключові слова: ґраткові моделі, групові розвинення, одночастинковий ґратковий потенціал, вільна енергія 